Historic, Archive Document

Do not assume content reflects current scientific knowledge, policies, or practices. 



\section{Wholesale Price List of Gladiolus SEASON 1930}

\section{Arthur A. Arenius 59 Bliss Road Longmeaclow, Mass.}

cre

CASH WITH ORDER - No orders taken for bulblets after April 1st 25 at 100 rate. 1000 at 8 time, 100 rate. 250 at 1000 rate.

Albania, fine white...

Anna Eberius, purple

Aviatrix, tall, buff shade

Bevier, salmon, cream throat

Break O'Day, early pink

Butterboy, straw yellow, early

Byron L. Smith, lavender

Chateau Thierry, large salmon red

Diana, red

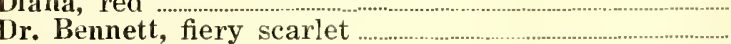

Dr. Neeley, tall pink

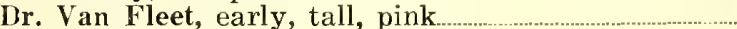

E. J. Shaylor,

Elaine, white

E. Tabor, very early, pink

Evelyn Kirtland, tall, pink

Fern Kyle, large, creamy white

Flaming Sword, tall, red

Giant Nymph, fine, pink

Gold Eagle, new, early, yellow

Golden Measure, large, yellow.

Halley, early, salmon pink

Henry Ford, large, purple.

Independence, pink

Indian Summer, late, lavender

Jewell, salmon pink

Kokomo, red, cream throat

Le M. Foch, early, flesh pink

Lilac Old Rose, color like name.

Lilac Wonder, lilac lavender

Lilywhite, early, white.

Longfellow, new, pink.

Los Angeles, tall, pink.

Maiden's Blush, very early, pink

Marie Kunderd, early, white

Meilust, early, salmon pink

Midsummer Dream, red.

Ming Toy, buff shade

Miss Spokane, late, red.

Mr. W. H. Phipps, prize-winning pink

Mrs. Dr. Norton, beautiful pink and cream.

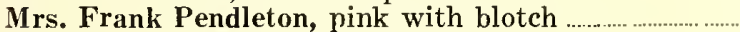

Mrs. Francis King, flame scarlet.

Mrs. L. S. Schweppe, pink with bloteh

Nimrod, new, early, pink...

Obelisque, creamy yellow

Odin, early, salmon pink

Opalescent, pale lavender

Orange Queen, orange

Primrose Beauty, creamy yellow.........

Purple Glory, dark maroon

Red Canna, tall, red

Salmon Buff, buff color.

Schwaben, yellow

Scarlet Beauty, scarlet

Scarlet Princeps, scarlet

Serratta, cream and yellow

Sheila, salmon pink

Souvenir, deep yellow

weet Lavender, early, lavender

Temblor, salmon pink

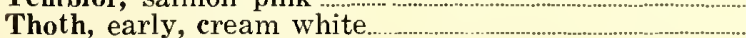

Tiffany, tall, pure white.

Topaz, salmon pink

Van der Neer, tall, purple

Vesta Tilley, white with blotch

Waneta, tall, pink

Willbrinck, early, flesh pink

Zanzibar, salmon pink.
Bulbs Per 100

Bulblets

\begin{tabular}{|c|c|c|c|c|c|c|c|}
\hline No. 1 & $\begin{array}{l}\text { No. } 2 \\
\$ 2.25\end{array}$ & $\begin{array}{l}\text { No. } 3 \\
\$ 2.00\end{array}$ & $\begin{array}{l}\text { No. } 4 \\
\$ 1.75\end{array}$ & $\begin{array}{l}\text { No.5 } \\
\$ 1.25\end{array}$ & $\begin{array}{l}\text { No. } 6 \\
\$ \quad .75\end{array}$ & $\begin{array}{c}1000 \\
\$ 1.00\end{array}$ & $\begin{array}{r}\text { Per Qt. } \\
\$ 3.00\end{array}$ \\
\hline$\$ 3.00$ & 2.50 & - & …….. & $\ldots \ldots$ & & & ............... \\
\hline 3.50 & 3.00 & & ................ & $\ldots \ldots$ & ........... & 1.00 & \\
\hline 2.50 & 2.00 & 1.50 & .............. & $\ldots \ldots \ldots$ & ........... & 2.00 & 5.00 \\
\hline 6.00 & 5.00 & 4.00 & ........... & $\ldots . . . . .$. & $\ldots$ & 2.00 & \\
\hline 2.00 & 1.75 & 1.50 & $\ldots . . \cdots \cdots$ & $\cdots \cdots$ & $\ldots \ldots$. & 1.00 & 2.50 \\
\hline 4.00 & $\begin{array}{l}4.00 \\
3.50\end{array}$ & $\begin{array}{l}3.00 \\
3.00\end{array}$ & 2.50 & 2.00 & 1.25 & $\begin{array}{l}2.00 \\
1.00\end{array}$ & 3.00 \\
\hline 3.00 & 2.50 & 2.00 & 1.50 & 1.00 & .50 & 1.00 & $\begin{array}{l}3.00 \\
3.00\end{array}$ \\
\hline 7.00 & 6.00 & 5.00 & 4.00 & 3.00 & 2.00 & 2.00 & \\
\hline 2.50 & 2.00 & 1.75 & 1.50 & 1.25 & 1.00 & .75 & 2.50 \\
\hline 3.00 & 2.50 & 2.00 & 1.50 & 1.00 & .75 & .50 & 2.00 \\
\hline 3.00 & 2.50 & 2.00 & 1.50 & 1.00 & .50 & .50 & 2.00 \\
\hline 5.00 & 4.00 & 3.00 & $\ldots . . . \cdots$ & ............ & .............. & 1.50 & 5.00 \\
\hline 4.00 & 3.25 & 2.75 & & & & 1.00 & 3.00 \\
\hline 3.00 & 2.50 & 2.00 & 1.50 & 1.00 & .75 & .50 & 2.00 \\
\hline 6.00 & 5.00 & 4.00 & 3.00 & 2.00 & 1.00 & 1.50 & 5.00 \\
\hline 3.00 & 2.50 & 2.00 & 1.50 & 1.00 & .75 & 1.00 & 3.00 \\
\hline 3.00 & 2.50 & 2.00 & 1.50 & 1.00 & .50 & 1.00 & 2.50 \\
\hline 4.50 & $\begin{array}{l}8.00 \\
4.00\end{array}$ & 7.00 & 6.00 & 4.00 & 2.00 & 5.00 & ............... \\
\hline 2.00 & 1.75 & 1.50 & 1.00 & .75 & .50 & .50 & 1.50 \\
\hline 250 & 200 & 2.50 & 2.00 & 1.50 & 1.00 & 1.00 & 3.00 \\
\hline 6.00 & 5.00 & 4.00 & 3.00 & 2.00 & 1.00 & 4.00 & 8.00 \\
\hline 2.50 & 2.00 & 1.50 & & & & .50 & 2.00 \\
\hline 4.00 & 3.25 & 2.50 & 2.00 & 1.50 & 1.00 & 1.00 & 3.50 \\
\hline 2.00 & 1.75 & 1.50 & & & & .50 & 2.00 \\
\hline 3.00 & 2.50 & 2.00 & 1.50 & 1.00 & .75 & 1.00 & 3.00 \\
\hline 3.00 & 2.50 & 2.00 & 1.50 & 1.00 & .75 & 1.00 & 3.00 \\
\hline 2.50 & 2.00 & & & & & 1.00 & 2.50 \\
\hline 8.00 & 7.00 & 6.00 & 5.00 & 4.00 & 3.00 & 3.00 & \\
\hline 3.00 & 2.50 & 2.00 & 1.50 & 1.00 & .50 & 1.50 & 4.00 \\
\hline 2.00 & 1.75 & 1.50 & 1.25 & .75 & .50 & .50 & 2.00 \\
\hline 3.50 & 3.00 & 2.50 & 2.00 & 1.50 & 1.00 & 1.00 & 4.00 \\
\hline 3.00 & 2.50 & 2.00 & 1.50 & 1.00 & .75 & 1.00 & 3.00 \\
\hline 3.00 & 2.50 & 2.00 & 1.50 & $\ldots$ & ........... & .50 & 2.00 \\
\hline 3.00 & 2.50 & 2.00 & & & & .50 & 2.00 \\
\hline 3.50 & 3.00 & 2.50 & 2.00 & 1.50 & 1.00 & 1.00 & 3.00 \\
\hline 9.00 & 8.00 & 6.50 & 5.00 & 4.00 & 2.00 & 2.50 & \\
\hline 2.75 & 2.25 & 1.75 & 1.25 & .75 & .50 & 1.00 & 2.50 \\
\hline & 2.50 & 2.00 & 1.50 & 1.00 & .50 & 1.00 & 2.50 \\
\hline 2.50 & 2.00 & & & & & 1.00 & 2.00 \\
\hline & & 2.00 & 1.50 & 1.00 & .75 & 1.00 & 3.00 \\
\hline 15.00 & 12.00 & 9.00 & & & & 5.00 & .......... \\
\hline 15.00 & 12.00 & 9.00 & 6.00 & 4.00 & 2.00 & 5.00 & .......... \\
\hline 3.50 & 3.00 & 2.50 & & & & 2.00 & \\
\hline 3.00 & 2.50 & 2.00 & 1.50 & 1.00 & .75 & 1.00 & 3.00 \\
\hline 5.00 & 4.25 & 3.50 & 2.75 & 2.25 & 2.00 & .75 & \\
\hline 3.00 & 2.50 & 2.00 & 1.50 & 1.00 & .75 & 1.00 & 3.00 \\
\hline 5.00 & 4.00 & & 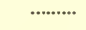 & ........ & ......" & 100 & \\
\hline 3.00 & 2.50 & 2.00 & & & 50 & 1.00 & 3.00 \\
\hline 2.50 & 2.25 & 1.75 & 1.25 & .75 & .50 & 1.00 & 2.50 \\
\hline 2.50 & 2.00 & & & & & & \\
\hline 3.00 & 2.50 & 2.00 & 1.50 & 1.00 & .50 & 1.00 & 3.00 \\
\hline & & 2.00 & 1.50 & 1.00 & .50 & 1.00 & 3.00 \\
\hline 5.00 & 4.00 & 3.00 & 2.00 & 1.00 & .50 & 1.50 & 5.00 \\
\hline 3.00 & 2.50 & 2.00 & 1.50 & 1.00 & .75 & 1.00 & 2.00 \\
\hline & & 2.00 & 1.50 & 1.00 & .75 & 1.00 & 3.00 \\
\hline 3.50 & 3.00 & 2.50 & 2.00 & 1.50 & 1.00 & 1.00 & 3.00 \\
\hline 3.00 & 2.50 & 2.00 & 1.50 & 1.00 & .50 & 1.00 & 3.00 \\
\hline 3.00 & 2.50 & 2.00 & 1.50 & 1.00 & .50 & 1.00 & 2.50 \\
\hline & 2.50 & 2.00 & 1.50 & 1.00 & .75 & 1.00 & 2.50 \\
\hline 3.00 & 2.50 & 2.00 & 1.50 & & 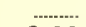 & .50 & 2.00 \\
\hline 7.00 & 6.00 & 5.00 & 4.00 & 3.00 & 2.00 & 1.50 & 5.00 \\
\hline 4.00 & 3.50 & 3.00 & 2.50 & 2.00 & 1.50 & 1.50 & 5.00 \\
\hline & 2.50 & 2.00 & & & & 1.00 & 3.00 \\
\hline 2.00 & 1.75 & 1.50 & 1.25 & 1.00 & .50 & .50 & 2.00 \\
\hline 5.00 & 4.00 & 3.00 & 2.00 & 1.00 & .50 & 1.00 & 3.00 \\
\hline
\end{tabular}

In order to make room for my seedlings I am selling outright many varieties at your own price. Write me. 
